\title{
EQUÍLIBRIO SÓLIDO-LÍQUIDO EM SISTEMAS MULTIFÁSICOS
}

\author{
F.UCCELLA, A. BERNARDO e M.GIULIETTI (in memorian)
}

Universidade Federal de São Carlos, Departamento de Engenharia Química

E-mail para contato: fernanda.uccella@gmail.com, abernardo@ufscar.br

\begin{abstract}
RESUMO - O estudo do equilíbrio sólido-líquido tem grande importância na indústria química, uma vez que está relacionado a processos de separação. Este experimento analisa a solubilidade e a cristalização do sal cloreto de potássio em sistemas multifásicos compostos por diferentes misturas de solventes. Por meio dos dados obtidos analisou-se os efeitos da temperatura, do etanol e do sal $\mathrm{MgCl}_{2}$ na solubilidade. Conclui-se que há uma maior quantidade de sal dissolvido conforme aumenta-se a temperatura e que maiores proporções de etanol e $\mathrm{MgCl}_{2}$ aumentam a precipitação do soluto.
\end{abstract}

\section{INTRODUÇÃO}

A termodinâmica do equilíbrio de fases de sistemas multifásicos é muito estudada para a otimização de processos industriais. O equilíbrio sólido-líquido, em que se desconsidera a fase vapor, é a base para processos de separação, como a cristalização. Esta operação é importante em processos industriais, uma vez que muitos produtos químicos são comercializados na fase sólida e devem ter elevada pureza, como na indústria farmacêutica e alimentícia.

O processo de cristalização é uma importante operação unitária de separação que baseia-se nas transferências de massa e quantidade de movimento. A supersaturação é a força motriz do processo, ou seja, ele ocorre em uma solução que contém uma quantidade de soluto maior do que a solubilidade. A cristalização divide-se em duas etapas: a nucleação, que é a formação dos cristais por meio da reorganização do soluto e o crescimento dos cristais. (Giulietti, et al. 2001)

A solubilidade é uma propriedade física de cada substância que determina a quantidade máxima de soluto que pode ser dissolvida em um solvente ou mistura de solventes, e depende da natureza das espécies envolvidas e da temperatura do sistema. Quando adiciona-se ao solvente a quantidade máxima de soluto, o sistema fica saturado e há equilíbrio entre as fases sólida e líquida. Para que ocorra a cristalização, é necessário que o sistema esteja supersaturado, ou seja, a concentração de soluto ultrapassa a quantidade de saturação. (Rios da Silva, 2010)

Segundo Myerson (2001), a curva de solubilidade pode ser melhor representada por uma variante do método isotérmico, no qual se acrescenta a agitação. Esse método consiste em adicionar o soluto em excesso ao solvente em um reator encamisado de temperatura constante e controlada, que deve ser mantido em agitação por no mínimo 4 horas e máximo 
de 24 horas. Após este período recolher-se uma amostra da mistura e mede-se a concentração de soluto. Repete-se o processo em diferentes temperaturas para a determinação da curva de solubilidade.

A modelagem termodinâmica de dados do equilíbrio sólido-líquido pode ser realizada pelos modelos semi-empíricos de van’t Hoff e de Apelblat, para uma melhor compreensão do processo de cristalização. Nestes modelos utiliza-se os dados coletados de fração molar do soluto e temperatura do sistema para a determinação do grau de não idealidade da solução saturada. (Uematsu, 2007)

A maioria dos sais possuem uma ampla e conhecida variação de solubilidade, considerando-se água como solvente, que geralmente cresce com o aumento de temperatura. No entanto, na indústria, para o desenvolvimento de um processo de cristalização para um soluto em particular, um solvente puro não é suficiente. Dessa forma, o objetivo deste trabalho é estudar a solubilidade e cristalização de um sal inorgânico em diferentes solventes.

\section{METODOLOGIA}

O estudo dos diferentes sistemas analisados neste experimento, iniciou-se com a construção de uma curva de solubilidade. Esta curva foi construída pelo aquecimento de 100 $\mathrm{g}$ de solvente em um reator encamisado até a temperatura desejada, por meio de um banho termostático. Em seguida, adicionou-se pequenas quantidades em massa de sal. Quando a solução se tornava homogênea, ou seja, quando o sal se dissolvia completamente no solvente, media-se o índice de refração por meio de um refratômetro. Esse procedimento foi realizado até que o soluto não se dissolvesse mais na mistura. Com os dados obtidos, plotou-se um gráfico do índice de refração em função da concentração de soluto na solução, esperando-se obter um perfil linear. A partir da equação da reta determinada foi possível calcular a solubilidade do sal em diferentes sistemas.

$\mathrm{Na}$ primeira etapa deste trabalho verificou-se a solubilidade de $\mathrm{KCl}$ em água para as temperaturas de $0^{\circ} \mathrm{C}, 25^{\circ} \mathrm{C}, 45^{\circ} \mathrm{C}$ e $65^{\circ} \mathrm{C}$. Após a construção da curva de calibração e a determinação da equação da reta, preparou-se novamente o sistema com o aquecimento de $100 \mathrm{~g}$ água destilada até a temperatura de estudo e posterior adição de uma massa superior a quantidade de sal não dissolvida na curva de calibração. A solução foi agitada por 24 horas e então mediu-se o índice de refração. Com este valor e a equação da reta determinada, calculou-se a solubilidade do sistema.

$\mathrm{O}$ mesmo procedimento foi realizado para o sistema $\mathrm{KCl}$ (soluto) em água e etanol (mistura de solventes). A concentração de etanol foi variada em $10 \%, 30 \%$ e $50 \%$ de massa de água nas temperaturas de $25^{\circ} \mathrm{C}, 45^{\circ} \mathrm{C}$ e $65^{\circ} \mathrm{C}$.

A solubilidade de $\mathrm{KCl}$ também foi determinada em uma mistura de solventes composta por água e $\mathrm{MgCl}_{2}$ hidratado $\left(\mathrm{MgCl}_{2} \cdot 6 \mathrm{H}_{2} \mathrm{O}\right)$. Assim, realizou-se cálculos para determinar a massa de sal hidratado que deveria ser usada para que a proporção do sal anidro fosse de $10 \%$, $15 \%, 20 \%$ e $25 \%$. Analogamente foi preparado em um reator uma mistura de $100 \mathrm{~g}$ de solvente e o experimento foi realizado nas temperaturas de $25^{\circ} \mathrm{C}, 45^{\circ} \mathrm{C}$ e $65^{\circ} \mathrm{C}$ sob agitação. Dessa forma, determinou-se a solubilidade de $\mathrm{KCl}$. 


\section{RESULTADOS}

\subsection{Equação de Van’t Hoff}

A análise dos dados obtidos neste experimento foi feita por meio do modelo semiempírico de Van’t Hoff, que está representada pela Equação 1.

$$
\ln (\gamma \cdot x)=\frac{\Delta H_{\text {fus̊̊̃o }}}{R} \cdot\left(\frac{1}{T_{\text {fus̊ีo }}}-\frac{1}{T}\right)
$$

Sendo $\gamma$ o coeficiente de atividade, $\mathrm{x}$ a fração molar do sal solúvel na solução, $\mathrm{R}$ a constante dos gases ideais, $\mathrm{T}$ a temperatura absoluta em kelvin da solução, $\Delta \mathrm{H}$ a entalpia de fusão e $T_{f}$ a temperatura de fusão do sal.

Quanto mais perto de 1 for o coeficiente de atividade, mais próxima da idealidade está a solução. Na Tabela 1 estão os dados de solubilidade do sal $\mathrm{KCl}$ (cloreto de potássio) em diferentes solventes, assim como os graus de idealidade correspondente, sendo a entalpia de fusão e temperatura de fusão iguais a $6400 \mathrm{cal} / \mathrm{mol} \mathrm{e} 773^{\circ} \mathrm{C}$. (Douglas; Harman, 1974)

Para os cálculos utilizou-se o valor de 1,987 cal/mol.K para a constante dos gases ideais.

Tabela 1 -Dados da solubilidade do $\mathrm{KCl}$ e do coeficiente de atividade da solução obtidos em diferentes misturas de solvente e temperatura

\begin{tabular}{|c|c|c|c|c|}
\hline $\begin{array}{c}\text { Temperatura } \\
(\mathrm{K})\end{array}$ & $\begin{array}{c}\text { Etanol } \\
(\% \text { massa })\end{array}$ & $\begin{array}{c}\mathrm{MgCl}_{2} \\
(\% \text { massa })\end{array}$ & $\begin{array}{c}\text { Fração molar } \\
\mathrm{KCl}\end{array}$ & $\begin{array}{c}\text { Coeficiente de } \\
\text { atividade }\end{array}$ \\
\hline 273,15 & 0 & 0 & 0,067540612 & $2,43 \mathrm{E}-03$ \\
\hline 298,15 & 0 & 0 & 0,073788800 & $5,99 \mathrm{E}-03$ \\
\hline 298,15 & 10 & 0 & 0,062659760 & $7,05 \mathrm{E}-03$ \\
\hline 298,15 & 30 & 0 & 0,036987693 & $1,19 \mathrm{E}-02$ \\
\hline 298,15 & 50 & 0 & 0,017064971 & $2,59 \mathrm{E}-02$ \\
\hline 298,15 & 0 & 10 & 0,048501518 & $9,11 \mathrm{E}-03$ \\
\hline 298,15 & 0 & 15 & 0,039325113 & $1,12 \mathrm{E}-02$ \\
\hline 298,15 & 0 & 20 & 0,026940089 & $1,64 \mathrm{E}-02$ \\
\hline 298,15 & 0 & 25 & 0,016292190 & $2,71 \mathrm{E}-02$ \\
\hline 318,15 & 0 & 0 & 0,092069480 & $9,47 \mathrm{E}-03$ \\
\hline 318,15 & 10 & 0 & 0,067156050 & $1,30 \mathrm{E}-02$ \\
\hline 318,15 & 30 & 0 & 0,036987693 & $2,36 \mathrm{E}-02$ \\
\hline 318,15 & 50 & 0 & 0,017064970 & $5,11 \mathrm{E}-02$ \\
\hline 318,15 & 0 & 10 & 0,056576367 & $1,54 \mathrm{E}-02$ \\
\hline 318,15 & 0 & 15 & 0,054805256 & $1,59 \mathrm{E}-02$ \\
\hline 318,15 & 0 & 20 & 0,026940089 & $3,24 \mathrm{E}-02$ \\
\hline 318,15 & 0 & 25 & 0,022987693 & $3,79 \mathrm{E}-02$ \\
\hline 338,15 & 0 & 0 & 0,099614926 & $1,59 \mathrm{E}-02$ \\
\hline 338,15 & 10 & 0 & 0,084717972 & $1,87 \mathrm{E}-02$ \\
\hline 338,15 & 30 & 0 & 0,061031726 & $2,60 \mathrm{E}-02$ \\
\hline 338,15 & 50 & 0 & 0,040000285 & $3,97 \mathrm{E}-02$ \\
\hline
\end{tabular}




\subsection{Análise Qualitativa}

Para efeito de comparação foi analisado o efeito do etanol e do sal $\mathrm{MgCl}_{2}$ na solubilidade do $\mathrm{KCl}$.

A Figura 1 representa o gráfico comparativo entre as massas de $\mathrm{KCl}$ solúveis no sistema em diferentes misturas de solvente a $25^{\circ} \mathrm{C}$, assim como o valor encontrado na literatura da solubilidade deste sal em água. $\mathrm{O}$ solvente varia de água pura a misturas de solvente contendo diferentes proporções de etanol e de $\mathrm{MgCl}_{2}$. (Battino, 1981)

Figura 1 - Gráfico da solubilidade de $\mathrm{KCl}$ em diferentes misturas de solvente à $25^{\circ} \mathrm{C}$.

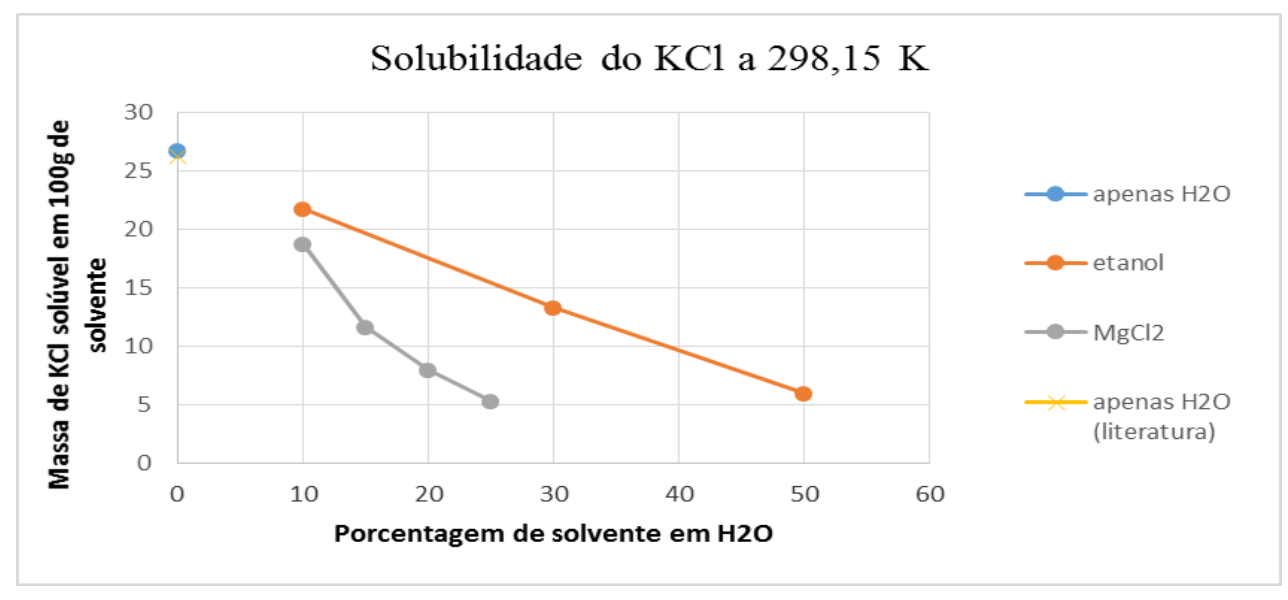

A Figura 2 e a Figura 3 também são gráficos comparativos do efeito das diferentes misturas de solvente, nas temperaturas de $45^{\circ} \mathrm{C}$ e $65^{\circ} \mathrm{C}$ respectivamente.

Figura 2 - Gráfico da solubilidade de $\mathrm{KCl}$ em diferentes misturas de solvente à $45^{\circ} \mathrm{C}$.

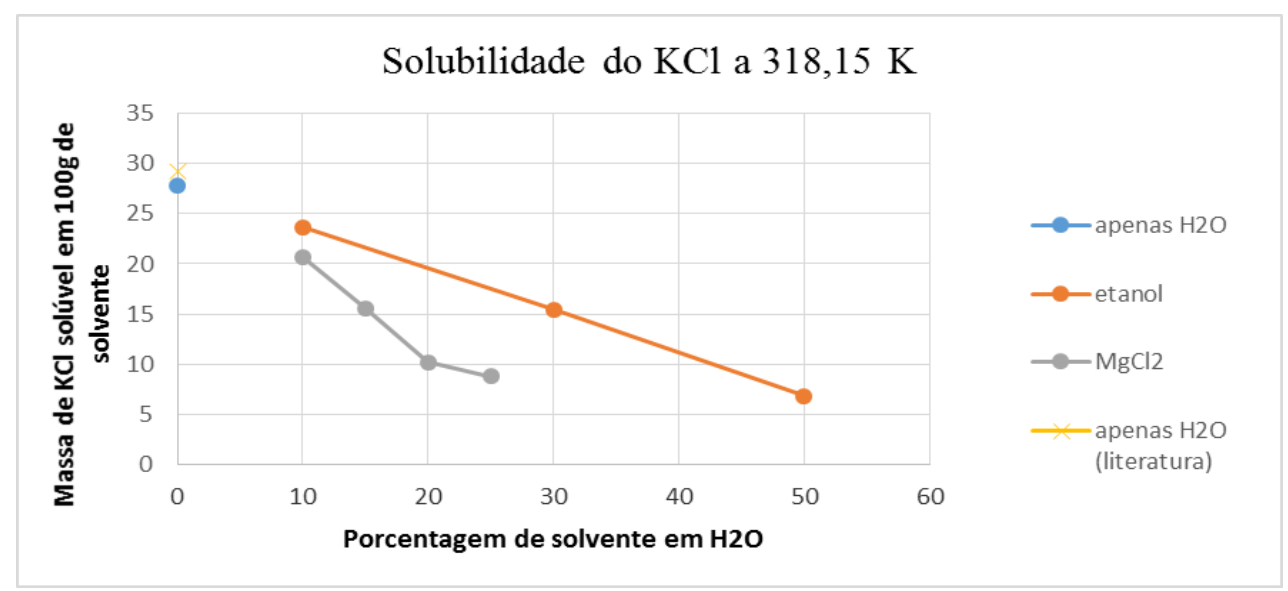


Figura 3 - Gráfico da solubilidade de $\mathrm{KCl}$ em diferentes misturas de solvente à $65^{\circ} \mathrm{C}$.

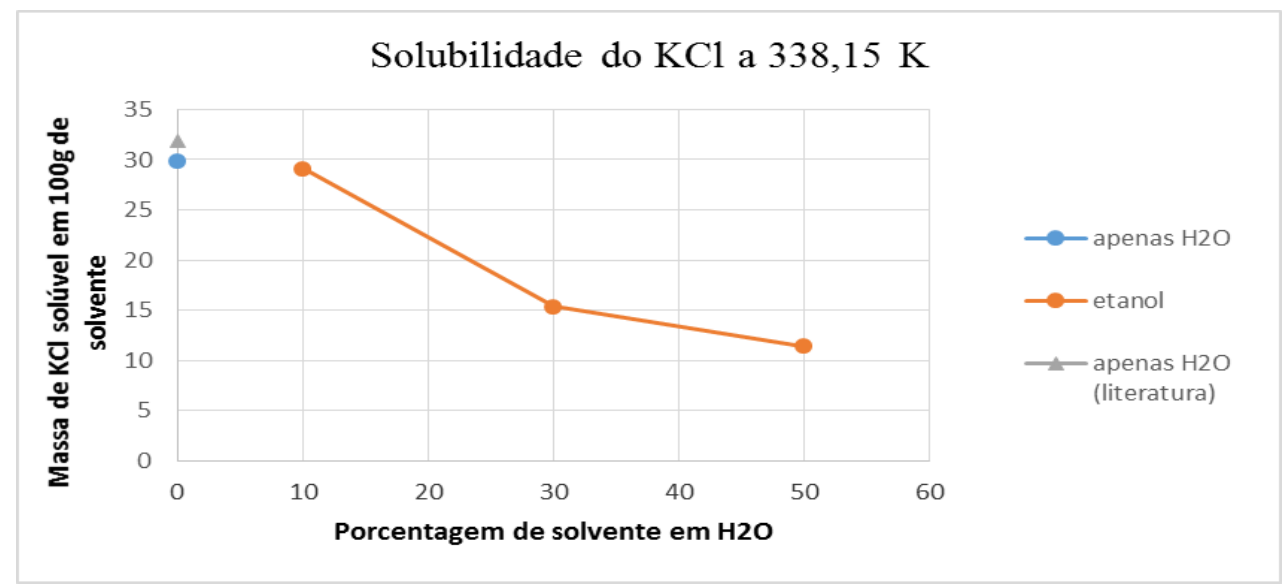

\section{DISCUSSÃO}

A partir da análise da Tabela 1 é possível observar que para todos os sistemas, quanto maior for a solubilidade, menor é o coeficiente de atividade. Dessa forma, os sistemas que proporcionam uma maior solubilidade ao sal são aqueles que mais se afastam da idealidade, ou seja, possuem uma maior fugacidade. Isto ocorre porque a idealidade de uma solução está relacionada à concentração de soluto.

Além disso é possível analisar o efeito da temperatura na solubilidade. Independente da mistura de solventes utilizada, a solubilidade cresce com o aumento da temperatura e, consequentemente, diminui-se o coeficiente de atividade.

O efeito da temperatura também é observado nas Figura 1, Figura 2 e Figura 3. Os três gráficos representam a solubilidade do sal cloreto de potássio e ao compará-los é possível afirmar que a massa de $\mathrm{KCl}$ solúvel em 100 gramas de solvente é maior na temperatura de $338,15 \mathrm{~K}$ do que em $318,15 \mathrm{~K}$, e a solubilidade nesta temperatura é maior que em 298,15 K.

No sistema formado por uma mistura de solventes composta por água e cloreto de magnésio a 338,15 K, não se calculou a solubilidade do cloreto de potássio, uma vez que não foi possível medir o índice de refração da solução. Durante o experimento, o $\mathrm{KCl}$ adicionado à mistura de solventes foi dissolvido, mas ao se retirar uma alíquota da solução que estava na temperatura do sistema, houve cristalização, impedindo a medida do índice de refração. Em temperaturas elevadas sob agitação, a solubilidade do sal aumenta, existindo mais soluto dissolvido do que a capacidade da solução, criando-se uma solução supersaturada. Por ser uma condição muito instável, ocorreu cristalização quando pipetou-se uma amostra.

Com base na Figura 1, Figura 2 e Figura 3 também foi possível analisar o efeito do etanol na solubilidade do $\mathrm{KCl}$. Para as três temperaturas estudadas, as misturas de solvente diminuíram a solubilidade do cloreto de potássio, quanto maior a porcentagem de etanol, maior a precipitação. Esse efeito é causado já que água e álcoois possuem afinidade e, assim, a água deixa de dissolver o sal para interagir com as moléculas de etanol. 
A solubilidade do cloreto de potássio também é diminuída quando a mistura de solvente é composta por água e outro sal, no caso $\mathrm{MgCl}_{2}$, como representa a Figura 1 e Figura 2. A precipitação do $\mathrm{KCl}$ aumenta, uma vez que o equilíbrio de fases da mistura é alterado pelo "efeito sal" ou salting out. A presença de um sal dissolvido como solvente aumenta a concentração da solução, pois este sal atrai as moléculas de água, que seriam responsáveis pela dissolução do $\mathrm{KCl}$.

Além disso, as figuras indicam que a solubilidade em água do cloreto de potássio obtida no experimento é muito próxima dos valores encontrados na literatura. A pequena diferença entre os valores é causada por erros aleatórios e sistemáticos. Os equipamentos utilizados possuem incertezas que interferem no valor final, como também as variáveis incontroláveis do laboratório.

\section{CONCLUSÃO}

$\mathrm{Na}$ indústria, o estudo do equilíbrio sólido-líquido em sistemas multifásicos é importante para o aperfeiçoamento de técnicas de separação. Com este trabalho conclui-se que a solubilidade de um soluto aumenta ou diminui de acordo com a variação de alguns fatores.

O aumento de temperatura possibilita uma maior quantidade de soluto dissolvida na solução e, consequentemente, a solução possui uma maior fugacidade, ou seja, há um maior afastamento da idealidade. Já a cristalização do soluto é proporcionada pela utilização de misturas de solvente contendo etanol e, ou sais dissolvidos, uma vez que estes interagem com as moléculas de água que seriam responsáveis pela dissolução do soluto.

\section{REFERÊNCIAS}

BATTINO, R.; Solubility data series, Dayton: Pergamon Press, 1981.

DOUGLAS, T. B.; HARMAN, A. W.; Measured enthalpy and of crystalline and derived liquid thermodynamic potassium chloride, properties KCL, from 271 to $1174 \mathrm{~K}$. Journal of Research of the National I Bureau of Standards- A. Physics and Chemistry, Washington, v. 78A, Abr 1974

GIULIETTI, M.; SECKLER, M. M.; DERENZO, S.; RÉ, M.I.; CEKINSKI, E. Industrial crystallization and precipitation from solutions: state of the technique. Brazilian Journal of Chemical Engineering, São Paulo, v. 18, Dec. 2001.

MYERSON, A.S.; Handbook of industrial crystallization, Boston: Butterworth-Heinemann, 2002.

RIOS DA SILVA, A.T.C. Estudo da cristalização de frutose em diferentes meios. 2010. Dissertação (Mestrado) - Curso de Engenharia Química, UFSCar, São Carlos, 2010

UEMATSU, M. M.; Estudo experimental e ajuste de modelos para previsão da solubilidade sólido-líquido no Sistema ácido salicílico-etanol-água. 2007. Dissertação (Mestrado) Curso de Engenharia Química, USP, São Paulo, 2007. 